\title{
Comparison of the T2 Relaxation Time of the Temporomandibular Joint Articular Disk between Patients with Temporomandibular Disorders and Asymptomatic Volunteers
}

\author{
N. Kakimoto, H. Shimamoto, J. Chindasombatjaroen, T. Tsujimoto, S. Tomita, Y. Hasegawa, S. Murakami, and S. Furukawa
}

\begin{abstract}
BACKGROUND AND PURPOSE: T2 relaxation time is a quantitative MR imaging parameter used to detect degenerated cartilage in the knee and lumbar intervertebral disks. We measured the T2 relaxation time of the articular disk of the temporomandibular joint in patients with temporomandibular disorders and asymptomatic volunteers to demonstrate an association between $\mathrm{T} 2$ relaxation time and temporomandibular disorder MR imaging findings.
\end{abstract}

MATERIALS AND METHODS: One hundred forty-four patients with temporomandibular disorders and 17 volunteers were enrolled in this study. An 8-echo spin-echo sequence for measuring the T2 relaxation times was performed in the closed mouth position, and the T2 relaxation time of the entire articular disk was measured. Patients were classified according to the articular disk location and function, articular disk configuration, presence of joint effusion, osteoarthritis, and bone marrow abnormalities.

RESULTS: The T2 relaxation time of the entire articular disk was $29.3 \pm 3.8 \mathrm{~ms}$ in the volunteer group and $30.7 \pm 5.1 \mathrm{~ms}$ in the patient group $(P=.177)$. When subgroups were analyzed, however, the T2 relaxation times of the entire articular disk in the anterior disk displacement without reduction group, the marked or extensive joint effusion group, the osteoarthritis-positive group, and the bone marrow abnormality-positive group were significantly longer than those in the volunteer group $(P<.05)$.

CONCLUSIONS: The T2 relaxation times of the articular disk of the temporomandibular joint in patients with progressive temporomandibular disorders were longer than those of healthy volunteers.

ABBREVIATIONS: ADDWOR = anterior disk displacement without reduction; $\mathrm{ADDWR}=$ anterior disk displacement with reduction; $\mathrm{PADDWOR}=$ partial anterior disk displacement without reduction; PADDWR = partial anterior disk displacement with reduction; TMD = temporomandibular disorders; $T M J=$ temporomandibular joint

D isorders of the temporomandibular joint (TMJ) are characterized by intra-articular positional and/or structural abnormalities. ${ }^{1}$ MR imaging is the preferred imaging technique for diagnosing temporomandibular disorders (TMD). ${ }^{2}$ It has been reported that the diagnostic accuracy of MR imaging for the assessment of the articular disk position and articular disk formation is $95 \%$ and for the assessment of osseous changes is $93 \%$ in postmortem examinations. ${ }^{3}$ Numerous studies of TMD by using

Received October 23, 2013 accepted after revision December 11.

From the Department of Oral and Maxillofacial Radiology (N.K., H.S., T.T., S.T., S.M., S.F.), Osaka University Graduate School of Dentistry, Osaka, Japan; Department of Oral and Maxillofacial Radiology (J.C.), Faculty of Dentistry, Mahidol University, Bangkok, Thailand; and Department of Dentistry and Oral Surgery (Y.H.), Hyogo College of Medicine, Hyogo, Japan.

A part of this paper previously presented at: 18th International Congress of DentoMaxillo-Facial Radiology, May 25-29, 2011; Hiroshima, Japan.

Please address correspondence to Naoya Kakimoto, DDS, PhD, Department of Oral and Maxillofacial Radiology, Osaka University Graduate School of Dentistry, 1-8 Yamadaoka, Suita, Osaka 5650871, Japan; e-mail: kakimoto@dent.osaka-u.ac.jp http://dx.doi.org/10.3174/ajnr.A3880
MR imaging have reported qualitative and morphologic evaluations, because the most important subgroup of articular abnormalities in patients with TMD includes different forms of articular disk displacement and deformation of the articular disk, either with normal bony joint components or accompanied by degenerative joint diseases such as osteoarthritis. ${ }^{4}$ Other MR imaging findings of TMD, including joint effusion and bone marrow abnormalities, have also been evaluated qualitatively and morphologically., 5

The $\mathrm{T} 2$ relaxation time is a quantitative MR imaging parameter derived from multiecho spin-echo sequences. Measuring the T2 relaxation times by using MR imaging has been reported to detect degenerated cartilage in the knee and lumbar intervertebral disk. $^{7-11}$ The T2 relaxation time of the articular disk of the TMJ in healthy volunteers has been previously described by using a 3T MR imaging system, ${ }^{12}$ but the T2 relaxation time of the articular disk of the TMJ in patients with TMD has not been reported, to our knowledge.

We hypothesized that the $\mathrm{T} 2$ relaxation time of the articular 
disk of the TMJ correlates with the qualitative and morphologic variations in the pathology of the TMJ. The aims of this study were to measure the T2 relaxation time of the articular disk of the TMJ in patients with TMD and asymptomatic volunteers to demonstrate any association between $\mathrm{T} 2$ relaxation time and MR image findings of TMD.

\section{MATERIALS AND METHODS}

Institutional review board approval was obtained for this study, and all patients and volunteers provided their written informed consent after the nature of the procedure was fully explained.

\section{Study Population}

From 2009 to 2012, one hundred fifty patients with TMD referred for MR imaging due to facial or TMJ pain; mandibular dysfunction, such as clicking, crepitation, or locking; or suspected internal derangement were recruited for this study. Six patients were excluded from this study due to motion or metallic artifacts on MR images. After these exclusions, the remaining 144 patients (110 females, 34 males; median age, 36 years; age range, 11-80 years) were analyzed. In addition, 21 asymptomatic volunteers underwent MR imaging examinations. The MR images of the TMJ in 4 of these volunteers demonstrated abnormal findings such as anterior disk displacement; hence, data from these volunteers were excluded from this study. The remaining 17 asymptomatic healthy volunteers ( 5 women, 12 men; median age, 26 years; age range, 23-32 years) were considered to have normal TMJs.

\section{MR Imaging}

All subjects were examined by using a 1.5T MR imaging scanner (Signa HDxt 1.5T; GE Healthcare, Milwaukee, Wisconsin) equipped with a TMJ surface coil. The imaging protocol to diagnose TMD consisted of oblique sagittal and coronal fast spinecho proton-attenuation-weighted sequences (TR/TE/echo-train length/NEX, $2500 \mathrm{~ms} / 20 \mathrm{~ms} / 8 / 2$ ); fat-suppressed T2-weighted sequences (TR/TE/echo-train length/NEX, $2000 \mathrm{~ms} / 85 \mathrm{~ms} / 16 / 3$ ) obtained perpendicular and parallel to the long axis of the mandibular condyle in a closed-mouth position; and sagittal spinecho proton-attenuation-weighted sequences (TR/TE/echo-train length/NEX, $800 \mathrm{~ms} / 24 \mathrm{~ms} / 4 / 2$ ) in closed- and open-mouth positions with the following parameters: $120 \times 120 \mathrm{~mm}$ FOV; $256 \times$ 160 matrix size; 3 -mm section thickness; and 1-mm gap. In addition to these sequences, oblique sagittal 8-echo fast spin-echo sequences were obtained perpendicular to the long axis of the mandibular condyle in a closed-mouth position to measure the T2 relaxation times with the following parameters: TR/TE/NEX $=$ $1000 \mathrm{~ms} / 8.9,17.8,26.7,35.6,44.5,53.4,62.4,71.3 \mathrm{~ms} / 2 ; 120 \times$ $120 \mathrm{~mm}$ FOV; $256 \times 160$ matrix size; 4 -mm section thickness; and 1-mm gap. The total acquisition time for this T2 relaxation timemeasuring sequence was 5 minutes 22 seconds for each joint.

\section{MR Image Evaluation}

All MR images were independently evaluated by 2 oral and maxillofacial radiologists (N.K. with 16 years of experience and H.S. with 8 years of experience). In cases of disagreement, the final diagnosis was made by consensus. We evaluated the following features: the articular disk position and function, the articular disk configuration, joint effusion, osteoarthritis, and bone marrow abnormalities.

The articular disk position and function were classified into 5 categories, as reported by Tasaki et al, ${ }^{13}$ with some modifications: normal superior, partial anterior disk displacement with reduction (PADDWR), partial anterior disk displacement without reduction (PADDWOR), anterior disk displacement with reduction (ADDWR), or anterior disk displacement without reduction (ADDWOR) in closed- and open-mouth-position sagittal proton-attenuation-weighted MR images. The articular disk configuration was classified into 6 categories, as reported by Murakami et al, ${ }^{14}$ with some modifications: biconcave, biplanar, hemiconvex, thickening of the posterior band, biconvex, or folded on closed-mouth-position oblique sagittal proton-attenuationweighted MR images. Joint effusion was classified into 4 categories, as proposed by Larheim et $\mathrm{al}^{5}$ : no or minimal fluid, moderate fluid, marked fluid, or extensive fluid on closed-mouth-position oblique sagittal fat-suppressed T2-weighted MR images. Osteoarthritis was classified into negative and positive, in which condylar osteophytes or erosion was observed, as reported by Kirk $^{15}$ on closed-mouth-position oblique sagittal proton-attenuationweighted MR images. Bone marrow abnormalities of the mandibular condyle were classified into negative and positive on the basis of the presence of edema or osteonecrosis, as described by Larheim et $\mathrm{al}^{16}$ on closed-mouth-position oblique sagittal protonattenuation- and T2-weighted MR images.

\section{Measurement of the T2 Relaxation Time}

The 8-echo spin-echo image data were transferred to an independent workstation (Advantage Workstation, Version 4.4; GE Healthcare). ROIs for measuring the $\mathrm{T} 2$ relaxation time were manually placed by 2 observers independently (N.K. and H.S.) on the entire articular disk, the anterior band of the articular disk, and the posterior band of the articular disk (Fig 1). The T2 relaxation time of the articular disk was calculated by using a software program (Functool 4.4.5; GE Healthcare).

The average value measured by the 2 observers was defined as the T2 relaxation time. For the measurement of the intraobserver reproducibility, 1 observer (N.K.) placed ROIs on the entire articular disk in 17 healthy volunteers 10 times on different days.

\section{Statistical Analysis}

As mentioned above, the coefficient of variation of 10 datasets of the entire articular disk in healthy volunteers was calculated to evaluate the intraobserver reproducibility. To evaluate the interobserver reproducibility, the Pearson product moment correlation coefficient was calculated for the 2 observers' data for the T2 relaxation time of the entire articular disk in healthy volunteers and patients. In the healthy volunteers, a paired $t$ test was used to compare the $\mathrm{T} 2$ relaxation times of the entire articular disk between the right and left TMJs and between the anterior and posterior bands of the articular disk. A $P$ value $<.05$ was considered a significant difference. To compare the $\mathrm{T} 2$ relaxation times of the entire articular disk between healthy volunteers and patients, the Mann-Whitney $U$ test or the Kruskal Wallis test was performed. A $P$ value $<.05$ was considered a significant difference. A post hoc 

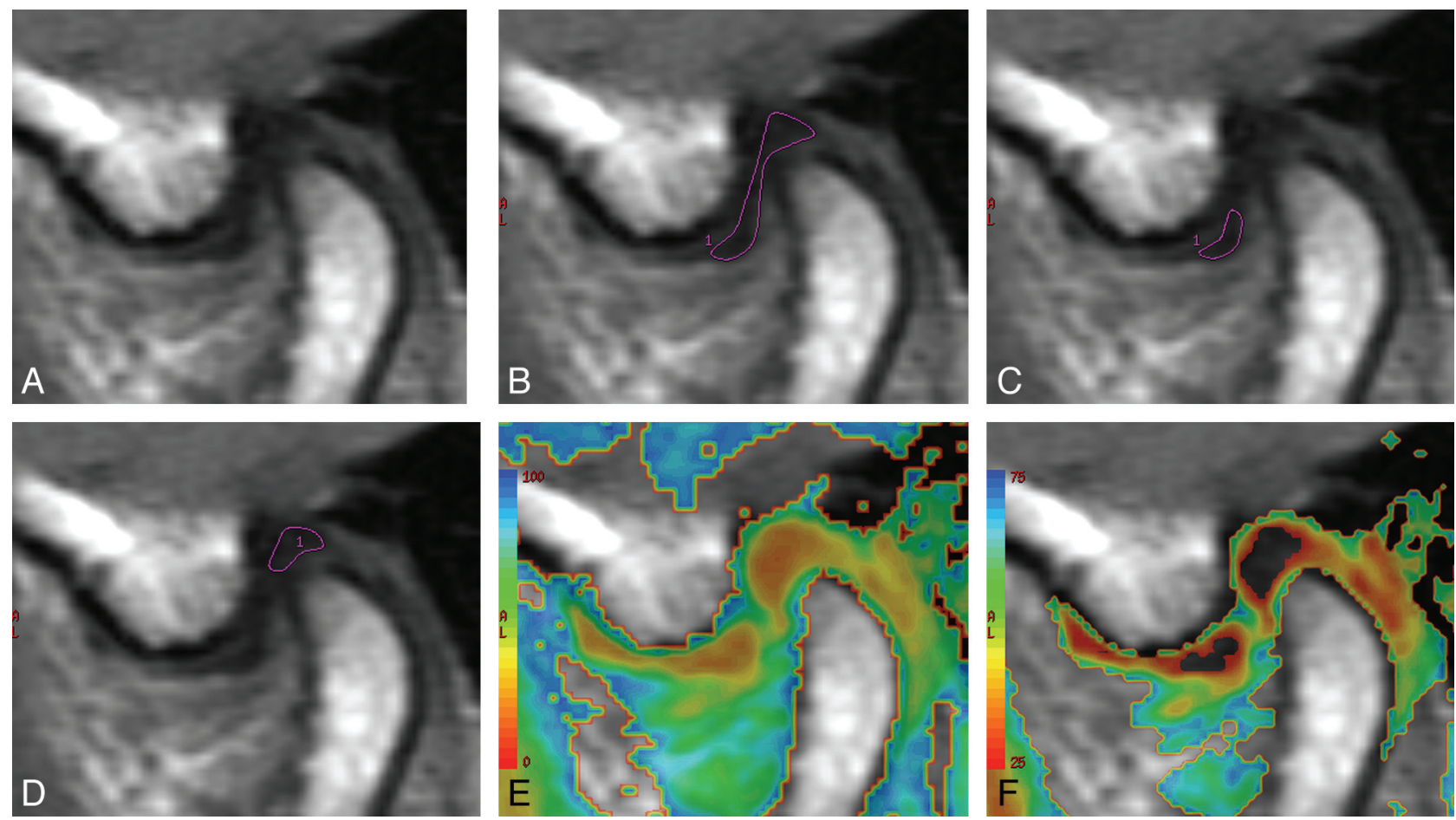

FIG 1. MR images of the articular disk of the TMJ, in a volunteer, used to measure the T2 relaxation time. $A$, The source MR image of the articular disk. The ROls used for the measurement of the T2 relaxation time of the entire articular disk (B), the anterior band of the articular disk $(C)$, and the posterior band of the articular disk $(D)$. The T2 relaxation times on a color map ranging from 0 to $100 \mathrm{~ms}(E)$ and 25 to $75 \mathrm{~ms}(F)$.

pair-wise analysis was performed by using the Mann-Whitney $U$ test with a Bonferroni correction, in which a $P$ value of $<.05 / 5$ for the articular disk position and function and joint effusion, .05/7 for the articular disk configuration, and .05/3 for osteoarthritis and bone marrow abnormalities was considered a significant difference. Multiple-regression analyses with simultaneous entry were used to identify important predictor variables for the T2 relaxation time of the entire disk among the MR image interpretations, and the patient age and sex were used as confounding variables. All of the statistical analyses were performed by using a commercially available software package (Statistical Package for the Social Sciences, Version 16.0; IBM, Armonk, New York).

\section{RESULTS}

The MR imaging findings of TMJs in healthy volunteers and patients are summarized in Table 1 . In the healthy volunteers, all joints showed normal articular disk position and function, and 32 biconcave and 2 biplanar disks were found. All joints were classified as having no or minimal fluid for joint effusion and were negative for both osteoarthritis and bone marrow abnormalities. In patients with TMD, most joints showed a normal articular disk position and function followed by anterior disk displacement without reduction, a biconcave disk, and no or minimal fluid for joint effusion and were negative for osteoarthritis and bone marrow abnormalities.

Intraobserver reproducibility by using the coefficient of variation of the T2 relaxation times of the entire articular disk in the healthy volunteers ranged from $1.1 \%$ to $4.7 \%$. Interobserver reproducibilities, determined by using the Pearson product moment correlation coefficient for the 2 observers' data for the entire

\section{Table 1: The patient and volunteer characteristics and MR} imaging findings

\begin{tabular}{|c|c|c|}
\hline & Volunteers & Patients \\
\hline Cases & 17 & 144 \\
\hline Male & 12 & 34 \\
\hline Female & 5 & 110 \\
\hline \multicolumn{3}{|l|}{ Age (yr) } \\
\hline Range & $23-32$ & $11-80$ \\
\hline Median & 26 & 36 \\
\hline \multicolumn{3}{|c|}{ Articular disk position and function (joints) } \\
\hline Normal superior & 34 & 113 \\
\hline PADDWR & 0 & 18 \\
\hline PADDWOR & 0 & 2 \\
\hline ADDWR & 0 & 48 \\
\hline ADDWOR & 0 & 107 \\
\hline \multicolumn{3}{|l|}{ Articular disk configuration (joints) } \\
\hline Biconcave & 32 & 143 \\
\hline Biplanar & 2 & 20 \\
\hline Hemiconvex & 0 & 37 \\
\hline Thickening of the posterior band & 0 & 36 \\
\hline Biconvex & 0 & 7 \\
\hline Folded & 0 & 45 \\
\hline \multicolumn{3}{|l|}{ Joint effusion (joints) } \\
\hline None or minimal fluid & 34 & 169 \\
\hline Moderate fluid & 0 & 80 \\
\hline Marked fluid & 0 & 24 \\
\hline Extensive fluid & 0 & 15 \\
\hline \multicolumn{3}{|l|}{ Osteoarthritis (joints) } \\
\hline Negative & 34 & 237 \\
\hline Positive & 0 & 51 \\
\hline \multicolumn{3}{|l|}{ Bone marrow abnormality (joints) } \\
\hline Negative & 34 & 260 \\
\hline Positive & 0 & 28 \\
\hline
\end{tabular}


Table 2: The $\mathrm{T} 2$ relaxation time of the articular disk

\begin{tabular}{lcc}
\hline & $\begin{array}{c}\text { T2 Relaxation } \\
\text { Time }(\mathbf{m s})\end{array}$ & $\boldsymbol{P}$ \\
\hline Volunteers & & \\
Left TMJ & $29.6 \pm 3.9$ & .585 \\
Right TMJ & $28.9 \pm 3.8$ & \\
All TMJs & $29.3 \pm 3.8$ & \\
Anterior band of all TMJs & $29.0 \pm 5.4$ & \\
Posterior band of all TMJs & $26.5 \pm 3.6$ & .007 \\
Male & $29.0 \pm 3.6$ & \\
Female & $29.8 \pm 4.3$ & .620 \\
Age $\leq 26$ years & $29.4 \pm 3.9$ & \\
Age $>26$ years & $29.1 \pm 3.8$ & .814 \\
Patients & & \\
All TMJs & $30.7 \pm 5.1$ & $.177^{\mathrm{a}}$ \\
Male & $29.5 \pm 4.0$ & \\
Female & $31.1 \pm 5.4$ & .890 \\
Age $\leq 36$ years & $29.5 \pm 4.4$ & \\
Age $>36$ years & $31.9 \pm 5.5$ & $<.001$ \\
\hline
\end{tabular}

${ }^{a}$ The T2 relaxation time of all TMJs in patients was compared with that of all TMJs in volunteers.

articular disk T2 relaxation time, were $0.862(P<.001)$ in the healthy volunteers and $0.891(P<.001)$ in patients.

The mean $\mathrm{T} 2$ relaxation times of the entire articular disk are shown in Table 2. In healthy volunteers, there was no significant difference in the T2 relaxation times of the entire disk between the left and right TMJs ( $29.6 \pm 3.9$ versus $28.9 \pm 3.8 \mathrm{~ms}, P=.585)$, whereas a significant difference between the $\mathrm{T} 2$ relaxation times of the anterior and posterior bands was observed $(29.0 \pm 5.4$ versus $26.5 \pm 3.6 \mathrm{~ms}, P=.007)$. Moreover, there were no significant differences in the $\mathrm{T} 2$ relaxation times of the entire articular disk between male and female groups $(29.0 \pm 3.6$ versus $29.8 \pm$ $4.3 \mathrm{~ms}, P=.620)$ and between younger and older age groups $(29.4 \pm 3.9$ versus $29.1 \pm 3.8 \mathrm{~ms}, P=.814)$. When healthy volunteers were compared with patients, the mean $\mathrm{T} 2$ relaxation times of the entire articular disk in healthy volunteers and patients with TMD were $29.3 \pm 3.8$ and $30.7 \pm 5.1 \mathrm{~ms}$, respectively. There were no significant differences in the mean $\mathrm{T} 2$ relaxation times of the entire articular disk between these 2 groups $(P=.177)$. In patients with TMD, there was no significant difference in the T2 relaxation time of the entire articular disk between male and female groups $(29.5 \pm 4.0$ versus $31.1 \pm 5.4 \mathrm{~ms}, P=.890)$. However, there was a significant difference in the T2 relaxation times of the entire disk between younger and older age groups $(29.5 \pm$ 4.4 versus $3.19 \pm 5.5 \mathrm{~ms}, P<.001)$.

With respect to the articular disk position and function, only 2 joints were classified as PADDWOR. The data from these 2 joints were excluded from further analyses of the articular disk position and function. The mean T2 relaxation times of the entire articular disk in the normal superior patients, the PADDWR, ADDWR, and ADDWOR groups were $29.4 \pm 4.2,28.9 \pm 4.0,30.5 \pm 5.3$, and $32.3 \pm 5.7 \mathrm{~ms}$, respectively. The mean $\mathrm{T} 2$ relaxation time of the entire articular disk in the ADDWOR group was significantly longer than that observed in the volunteer group $(P=.005)$ and the normal superior patient group $(P<.001)$ (Fig $2 A)$.

With respect to the articular disk configuration, the mean T2 relaxation times of the entire articular disk in the biconcave, biplanar, hemiconvex, thickening of the posterior band, biconvex, and folded groups were $30.0 \pm 4.6,29.9 \pm 4.8,32.5 \pm 6.6,30.7 \pm$
3.9, $31.6 \pm 6.9$, and $31.6 \pm 5.8 \mathrm{~ms}$, respectively. There were no significant differences among these groups (Fig $2 B$ ).

With respect to joint effusion, the mean $\mathrm{T} 2$ relaxation times of the entire articular disk in the patient groups with no or minimal fluid, moderate fluid, marked fluid, and extensive fluid were $30.0 \pm 4.7,30.2 \pm 4.5,34.0 \pm 6.3$, and $36.2 \pm 5.6 \mathrm{~ms}$, respectively. The mean T2 relaxation time of the entire articular disk in the marked or extensive fluid group was significantly longer than that observed in the healthy volunteer group $(P=.002$ or $P<.001)$, the patient group with no or minimal fluid $(P<.001$ or $P=.001)$, and the moderate fluid group $(P=.002$ or $P<.001)$ (Fig $2 C$ ).

With respect to osteoarthritis, the mean $\mathrm{T} 2$ relaxation times of the entire articular disk in patients with negative and positive findings were $29.9 \pm 4.3$ and $34.4 \pm 6.7 \mathrm{~ms}$, respectively. The mean T2 relaxation time of the entire articular disk in the osteoarthritis-positive group was significantly longer than that observed in the healthy volunteer group $(P<.001)$ and the osteoarthritis-negative group $(P<.001)$ (Fig $2 D)$.

With respect to bone marrow abnormalities, the mean T2 relaxation times of the entire articular disk in the patients with negative and positive findings were $30.4 \pm 5.0$ and $33.6 \pm 5.9 \mathrm{~ms}$, respectively. The mean $\mathrm{T} 2$ relaxation time of the entire articular disk in the bone marrow abnormality-positive group was significantly longer than that observed in the healthy volunteer group $(P=.003)$ and the bone marrow abnormality-negative in the group $(P=.002)$ (Fig $2 E)$.

According to the multiple-regression analysis, the important variables affecting the T2 relaxation time of the entire disk were identified as osteoarthritis $(P=.001)$, joint effusion $(P=.003)$, and bone marrow abnormalities $(P=.02)$ (Table 3$)$. The adjusted $R^{2}$ for the multiple regression analysis for the T2 relaxation time of the entire disk was $0.957(P<.001)$.

\section{DISCUSSION}

Quantitative measurements of the T2 relaxation time are useful for characterizing knee osteoarthritis and degenerated intervertebral disks. ${ }^{7-11}$ The immobilization of water protons in the cartilage by the collagen-proteoglycan matrix promotes $\mathrm{T} 2$ decay and renders the cartilage low in signal intensity on T2-weighted images, while mobile water protons in the synovial fluid retain their high signals. A loss of collagen and proteoglycan in degenerating cartilage increases the mobility of water, thus increasing its signal intensity on T2-weighted images. ${ }^{17}$ The T2 relaxation times of the intervertebral disk anulus fibrosus and nucleus pulposus correlate strongly with the water content and weakly with the proteoglycan content. ${ }^{18}$ It has been reported that the T2 relaxation times of the nucleus pulposus of the intervertebral disk in the lumbar spine exhibit significant differences in disks with herniation and anular tears compared with disks without these abnormalities, ${ }^{10}$ and the $\mathrm{T} 2$ relaxation time in lumbar disks was reported to be correlated with the stage of disk degeneration and patient age. ${ }^{11}$

Measurement of the T2 relaxation time of the TMJ articular disk in normal joints has only been performed in 1 previous study. ${ }^{12}$ In that study, the T2 relaxation time of the entire articular disk of the normal TMJ was $40.21 \pm 2.95 \mathrm{~ms}$, which was longer than the value measured in our study. They used a 3T MR imaging scanner to measure the $\mathrm{T} 2$ relaxation time of the articular disk, 


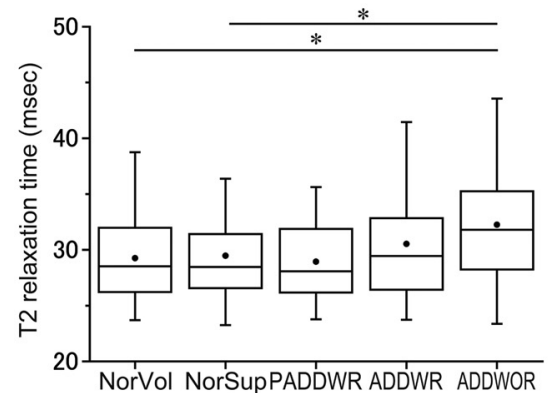

A

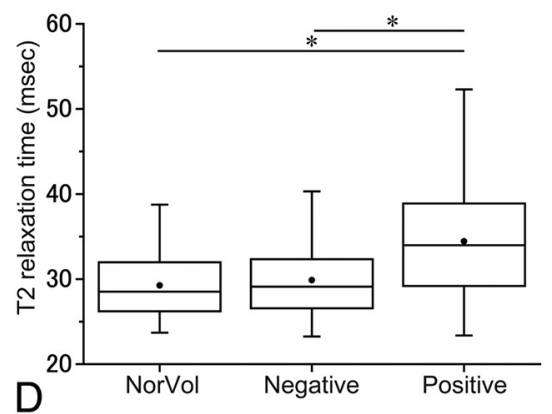

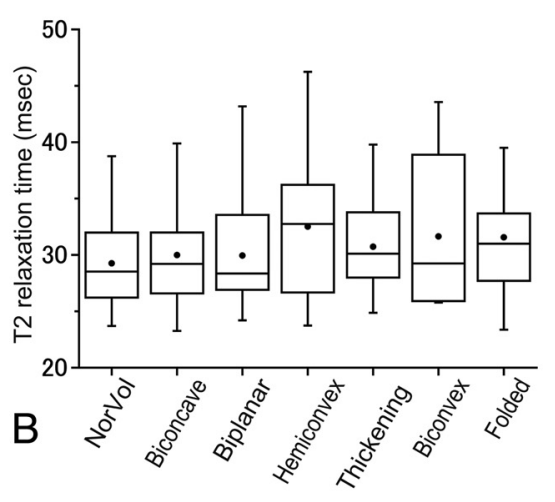

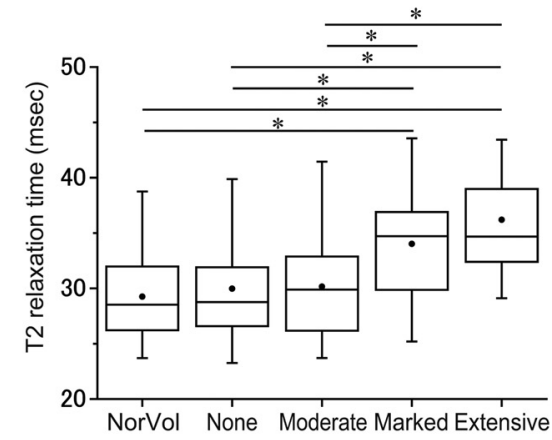

C

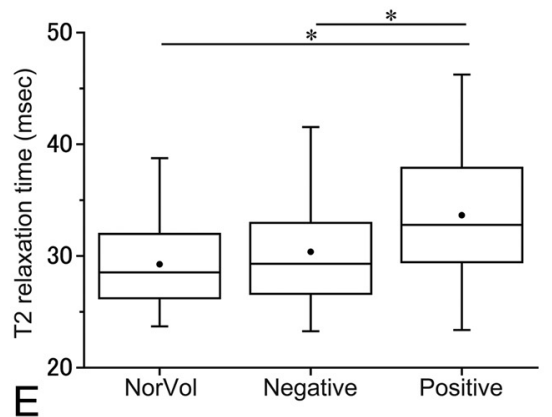

FIG 2. The $T 2$ relaxation times according to the MR image interpretations. $A$, The $T 2$ relaxation times according to the articular disk position and function categories. The asterisk indicates $P<.05 / 5$ in the Mann-Whitney test with a Bonferroni correction. $B$, The T2 relaxation times according to the articular disk configuration categories. "Thickening" indicates thickening of the posterior band. C, The T2 relaxation times according to the joint effusion categories. "None" indicates no or minimal fluid; "Moderate," moderate fluid; "Marked," marked fluid; and "Extensive," extensive fluid. The asterisk indicates $P<.05 / 5$ in the Mann-Whitney test with a Bonferroni correction. $D$, The T2 relaxation times according to the osteoarthritis categories. The asterisk indicates $P<.05 / 3$ in the Mann-Whitney test with a Bonferroni correction. $E$, The T2 relaxation times according to the bone marrow abnormality categories. The asterisk indicates $P<.05 / 3$ in the Mann-Whitney test with a Bonferroni correction. NorVol indicates healthy volunteers.

Table 3: The multiple regression model for $\mathrm{T} 2$ relaxation time of the entire disk

\begin{tabular}{|c|c|c|c|c|c|c|c|}
\hline \multirow{2}{*}{$\frac{\text { Variable }}{\text { Disk position and function }}$} & \multirow{2}{*}{$\begin{array}{c}\begin{array}{c}\text { Unstandardized } \\
\text { Coefficient (\$) }\end{array} \\
0.652\end{array}$} & \multirow{2}{*}{$\begin{array}{c}\begin{array}{c}\text { Unstandardized } \\
\text { Coefficient (SE) }\end{array} \\
1.116\end{array}$} & \multirow{2}{*}{$\begin{array}{c}\begin{array}{c}\text { Standardized } \\
\text { Coefficient (§) }\end{array} \\
0.052\end{array}$} & \multirow{2}{*}{$\begin{array}{c}T \\
0.585\end{array}$} & \multirow{2}{*}{$\begin{array}{c}P \\
.559\end{array}$} & \multicolumn{2}{|c|}{$\begin{array}{l}\text { 95\% Confidence } \\
\text { Interval }\end{array}$} \\
\hline & & & & & & -1.543 & 2.848 \\
\hline Disk configuration & -1.658 & 1.014 & -0.12 & -1.635 & .103 & -3.653 & 0.337 \\
\hline Joint effusion & 2.467 & 0.838 & 0.188 & 2.944 & .003 & 0.819 & 4.116 \\
\hline Osteoarthritis & 3.986 & 1.221 & 0.273 & 3.264 & .001 & 1.583 & 6.389 \\
\hline Bone marrow abnormalities & 2.896 & 1.243 & 0.19 & 2.331 & .02 & 0.451 & 5.341 \\
\hline
\end{tabular}

but we used a $1.5 \mathrm{~T}$ scanner. They also measured the T2 relaxation time at the anterior, middle, and posterior band and found that the middle zone of the articular disk showed longer T2 relaxation times than the anterior and posterior bands. In contrast to our study, there was no significant difference in the $\mathrm{T} 2$ relaxation times between the anterior and posterior bands. It has been reported that the elastic fiber attenuation of the anterior and posterior bands of the articular disk of the TMJ is significantly different. ${ }^{19}$ Therefore, the T2 relaxation time of both bands may be different, as shown in our study. Further investigations might be necessary to confirm which findings are appropriate and whether there are population-based differences or scanner magnetstrength differences. Moreover, in patients with TMD, the T2 relaxation time of the entire articular disk in the older age group was significantly longer than that in the younger age group. Further investigations are needed for the correlation of age or duration of TMD and the $\mathrm{T} 2$ relaxation time.
The TMJ articular disk is composed of collagen fibers, proteoglycans, and tissue fluid. ${ }^{20}$ It has been reported that the content of glycosaminoglycan, a polysaccharide attached to a core protein in proteoglycan, in the articular disk of the TMJ in patients with anterior disk displacement and those with chronic closed lock is considerably lower than that observed in normal disk tissue. ${ }^{21,22}$ Therefore, the composition of the articular disk is thought to change under the above conditions. In this study, although patients and healthy volunteers showed no differences in the T2 relaxation time of the entire articular disk, the T2 relaxation times of the entire articular disk in the ADDWOR group, the severe joint effusion group, the osteoarthritis-positive group, and the bone marrow abnormality-positive group were significantly longer than that observed in the volunteer group. The multiple regression analyses showed that osteoarthritis, joint effusion, and bone marrow abnormalities were important variables affecting the $\mathrm{T} 2$ relaxation time of the entire disk. This finding means that 
the T2 relaxation time of the articular disk is longer in patients with advanced TMD findings on MR imaging. Therefore, the T2 relaxation time of the articular disk of the TMJ may correlate with a progressive course of TMD.

It has been reported that the articular disk configuration changed from biconcave configuration to distorted configuration after disk displacement. ${ }^{14}$ Then the T2 relaxation time of the distorted articular disk may show a value similar to that of the T2 relaxation time of the ADDWOR group. In our study, the T2 relaxation times of the hemiconvex, thickening of the posterior band, biconvex, and folded group were $32.5 \pm 6.6$, $30.7 \pm 3.9$, $31.6 \pm 6.9$, and $31.6 \pm 5.8 \mathrm{~ms}$, respectively, and that of ADDWOR group was $32.3 \pm 5.7 \mathrm{~ms}$. These T2 relaxation times were fairly similar. Although there were no significant differences among the T2 relaxation times of these disk-configuration groups, further study is needed.

There are some limitations associated with the present study. First, the T2 relaxation times were only measured on the central portion of the articular disk. The medial and lateral areas of the articular disk could not be evaluated because the section thickness of the MR images used to measure the T2 relaxation time was set to $4 \mathrm{~mm}$ with a $1-\mathrm{mm}$ intersection gap, and this thickness led to a limited volume of the articular disk appearing on MR images. Second, there was no histopathologic correlation with the T2 relaxation time of the articular disk in this study. Almost all patients with TMD were treated conservatively with medication, splint therapy, or physical therapy, and due to the high diagnostic accuracy of MR imaging for assessing $\mathrm{TMD},{ }^{3}$ the conditions of patients were not confirmed histopathologically. On the basis our study, the potential alterations in the T2 relaxation time of the articular disk may result from underlying pathologic conditions; thus, the T2 relaxation time can provide important information about TMD. Third, there was no clinical correlation such as pain scale with the T2 relaxation time of the articular disk in this study. Further studies with clinical correlation are needed to confirm the significance of the $\mathrm{T} 2$ relaxation time.

\section{CONCLUSIONS}

The T2 relaxation time of the entire articular disk was $29.3 \pm 3.8$ $\mathrm{ms}$ in healthy volunteers and $30.7 \pm 5.1 \mathrm{~ms}$ in patients with TMD, and there was no significant difference between these groups. The T2 relaxation times of the entire articular disk in the ADDWOR group, the severe joint effusion group, the osteoarthritis-positive group, and the bone marrow abnormality-positive group were, however, significantly longer than those observed in the volunteer group; and the T2 relaxation times of the articular disk of the TMJ in patients with progressive TMD were longer than those of healthy volunteers. Hence, the T2 relaxation time of the TMJ articular disk may correlate with a progressive course of TMD.

\section{REFERENCES}

1. Tanaka E, Detamore MS, Mercuri LG. Degenerative disorders of the temporomandibular joint: etiology, diagnosis, and treatment. $J$ Dent Res 2008;87:296-307

2. Larheim TA, Westesson PL. TMJ imaging. In: Laskin DM, Greene CS,
Hylander WL, eds. Temporomandibular Disorders An Evidence-Based Approach to Diagnosis and Treatment. Chicago: Quintessence Publishing Co.; 2006:149-80

3. Tasaki MM, Westesson PL. Temporomandibular joint: diagnostic accuracy with sagittal and coronal MR imaging. Radiology 1993; 186:723-29

4. Larheim TA. Current trends in temporomandibular joint imaging. Oral Surg Oral Med Oral Pathol Oral Radiol Endod 1995;80:555-76

5. Larheim TA, Westesson PL, Sano T. MR grading of temporomandibular joint fluid: association with disk displacement categories, condyle marrow abnormalities and pain. Int J Oral Maxillofac Surg 2001;30:104-12

6. Sano T, Westesson PL, Larheim TA, et al. The association of temporomandibular joint pain with abnormal bone marrow in the mandibular condyle. J Oral Maxillofac Surg 2000;58:254-57, discussion 258-59

7. Dunn TC, Lu Y, Jin H, et al. T2 relaxation time of cartilage at MR imaging: comparison with severity of knee osteoarthritis. Radiology 2004;232:592-98

8. Koff MF, Amrami KK, Kaufman KR. Clinical evaluation of T2 values of patellar cartilage in patients with osteoarthritis. Osteoarthritis Cartilage 2007;15:198-204

9. Ludescher B, Effelsberg J, Martirosian P, et al. T2- and diffusionmaps reveal diurnal changes of intervertebral disc composition: an in vivo MRI study at 1.5 Tesla. J Magn Reson Imaging 2008;28:252-57

10. Trattnig S, Stelzeneder D, Goed S, et al. Lumbar intervertebral disc abnormalities: comparison of quantitative T2 mapping with conventional MR at 3.0 T. Eur Radiol 2010;20:2715-22

11. Marinelli NL, Haughton VM, Anderson PA. T2 relaxation times correlated with stage of lumbar intervertebral disk degeneration and patient age. AJNR Am J Neuroradiol 2010;31:1278-82

12. Cao Y, Xia C, Wang S, et al. Application of magnetic resonance T2 mapping in the temporomandibular joints. Oral Surg Oral Med Oral Pathol Oral Radiol 2012;114:644-49

13. Tasaki MM, Westesson PL, Isberg AM, et al. Classification and prevalence of temporomandibular joint disk displacement in patients and symptom-free volunteers. Am J Orthod Dentofacial Orthop 1996;109:249-62

14. Murakami S, Takahashi A, Nishiyama $H$, et al. Magnetic resonance evaluation of the temporomandibular joint disc position and configuration. Dentomaxillofac Radiol 1993;22:205-07

15. Kirk WS Jr. Sagittal magnetic resonance image characteristics and surgical findings of mandibular condyle surface disease in staged internal derangements. J Oral Maxillofac Surg 1994;52:64-68

16. Larheim TA, Westesson PL, Hicks DG, et al. Osteonecrosis of the temporomandibular joint: correlation of magnetic resonance imaging and histology. J Oral Maxillofac Surg 1999;57:888-98, discussion 899

17. Blumenkrantz G, Majumdar S. Quantitative magnetic resonance imaging of articular cartilage in osteoarthritis. Eur Cell Mater 2007;13:76-86

18. Marinelli NL, Haughton VM, Munoz A, et al. T2 relaxation times of intervertebral disc tissue correlated with water content and proteoglycan content. Spine 2009;34:520-24

19. Clément C, Bravetti P, Plenat F, et al. Quantitative analysis of the elastic fibres in the human temporomandibular articular disc and its attachments. Int J Oral Maxillofac Surg 2006;35:1120-26

20. Tanaka E, van Eijden T. Biomechanical behavior of the temporomandibular joint disc. Crit Rev Oral Biol Med 2003;14:138-50

21. Axelsson S, Holmlund A, Hjerpe A. Glycosaminoglycans in normal and osteoarthrotic human temporomandibular joint disks. Acta Odontol Scand 1992;50:113-19

22. Paegle DI, Holmlund AB, Hjerpe A. Matrix glycosaminoglycans in the temporomandibular joint in patients with painful clicking and chronic closed lock. Int J Oral Maxillofac Surg 2003;32:397-400 